\title{
Use of enhanced nucleation surfaces in a continuous flow crystallization system
}

Kyle Nordquist knordquist@denovx.com

Tiffany Kinnibrugh tkinnibrugh@denovx.com

Kevin Scaab kschaab@denovx.com

Andrew Bond abond@denovx.com

DeNovX, 3440 S. Dearborn St., Lab 204 S, Chicago, IL 60616

Cost effective and reproducible process scale crystallization plays an integral role in the production of over $90 \%$ of all active pharmaceutical ingredients (APIs). Despite advances to traditional batch manufacturing technologies, minor variations in the hydrodynamic conditions can dramatically affect crystal nucleation and can adversely impact average crystal sizes and size distributions. Batches of crystalline API that do not meet regulated sizing specifications may require additional sizing procedures (e.g., sieving, milling, etc.), which come with the economic penalties of lost time and material. DeNovX is continuing the development of a fume hoodsized continuous flow antisolvent crystallization system that can efficiently produce $5-10 \mathrm{~kg} /$ day of crystalline API having small average crystal sizes and/or narrow crystal size distributions. The device is compatible with enhanced nucleation features that alter the surface energy of the reaction vessel, which facilitates interactions with the solutes to give improved crystal nucleation. The enhanced nucleation surfaces coupled with the continuous flow conditions combine to give a potentially tunable approach for efficiently producing APIs in a compact footprint with small average crystal sizes and narrow crystal size distributions. Controlled, replicate studies with flufenamic acid as a benchmark API have roughly shown a $40 \%$ reduction in average crystal sizes from $82(31)$ to $51(36) \mu \mathrm{m}$ with an approximate $45 \%$ decrease in the overall crystal size distribution. In a challenge to the utility of the technique, a continuous flow antisolvent crystallization method was developed for the acetaminophen:2,4-

pyridinedicarboxylic acid cocrystalline material. In these challenge experiments, both crystalline precursors were successfully stabilized in the 2-propanol solvent prior to addition of the $n$ hexane antisolvent that generated the desired cocrystalline material, with chemical identities for each of the two crystalline reactants and sole crystalline product confirmed by powder X-ray diffraction. Controlled, replicate studies at $0.20 \mathrm{M}$ solute concentrations with enhanced nucleation surfaces and four $\mathrm{n}$-hexane antisolvent injection points produced $88 \%$ of the desired cocrystalline material in a notably narrow range of $16-28 \mu \mathrm{m}$. 